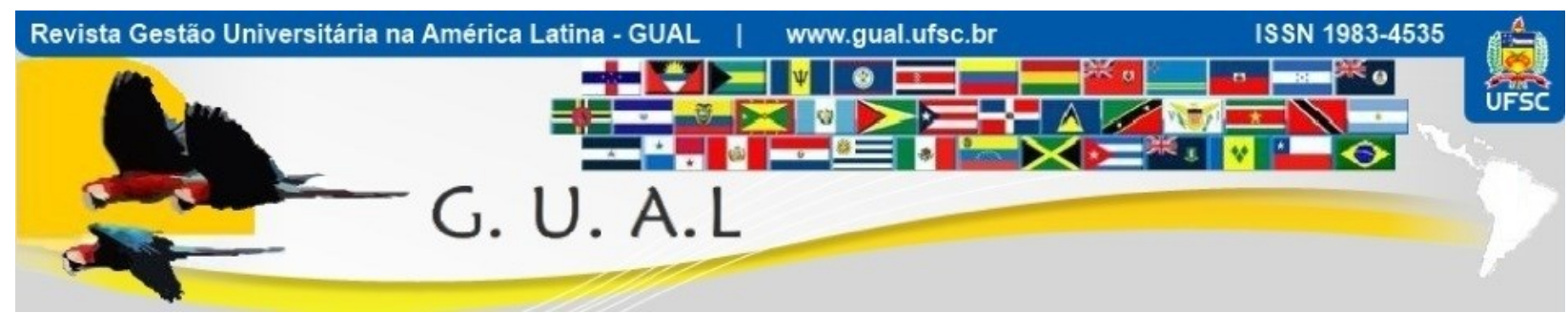

DOI: http://dx.doi.org/10.5007/1983-4535.2015v8n3p26

\title{
ANÁLISE DA ADAPTAÇÃO CULTURAL DE ESTUDANTES INTERNACIONAIS SOB O PONTO DE VISTA DAS TEORIAS DA CURVA "U" E DA CURVA "W"
}

\section{ANALYSIS OF CULTURAL ADAPTATION OF INTERNATIONAL STUDENTS UNDER THE POINT OF VIEW OF THE CURVE THEORY "U" AND "W"}

Luciane Stallivieri, Doutora Universidade Federal de Santa Catarina - UFSC $\underline{\text { luciane.stallivieri@ufsc.br }}$

Daísa Ziglioli Pilotto, Graduada Universidade de Caxias do Sul - UCS daisazp@,hotmail.com

Roberto Birch Gonçalves, Doutor Universidade de Caxias do Sul - UCS rbgoncal@ucs.br

Recebido em 10/julho/2014

Aprovado em 18/julho/2015

Sistema de Avaliação: Double Blind Review

Esta obra está sob uma Licença Creative Commons Atribuição-Uso. 


\title{
RESUMO
}

Estudantes de todo o mundo estão cada vez mais interessados em aprender novos idiomas e inserirem-se em culturas diferentes, intensificando os números da mobilidade acadêmica internacional. No entanto, quando estes estudantes viajam para outro país, as diferenças culturais são fortemente percebidas, mesmo que de formas diferentes, influenciando o processo de adaptação. Dada a relevância e o crescimento dos números da mobilidade acadêmica internacional, o presente estudo analisa o processo de adaptação cultural de estudantes em mobilidade, considerando os pressupostos das teorias das curvas "U" $\mathrm{e}$ "W". Quanto à metodologia, trata-se de uma pesquisa descritiva com abordagem quantitativa. A coleta de dados foi realizada através de questionários fechados enviados a estudantes internacionais de diferentes países que estudaram ou que ainda estão estudando numa Instituição de Ensino Superior brasileira. Os resultados mostram que, após a fase do choque cultural, os estudantes se sentem mais conectados à cultura local, fazem mais amizades e se sentem mais confiantes. É possível afirmar, também, que quase todos os estudantes internacionais conseguiram se adaptar bem aos costumes locais.

Palavras-chave: Mobilidade Acadêmica. Adaptação Cultural. Estudantes Internacionais. Curva "U" e "W".

\begin{abstract}
Students from around the world are increasingly interested in learning new languages and be inserted in different cultures, increasing the numbers of international mobility. However, when traveling to another country, cultural differences are strongly perceived, even if in different ways, influencing the adaptation process. Given the importance and growth of the international academic mobility, this study analyzes the process of cultural adaptation of students in mobility, considering the assumptions of the theory of curves " $U$ " and "W". As for the methodology, it is a descriptive research with quantitative approach. Data collection was conducted through closed questionnaires sent to international students from different countries who have studied or are still studying in a Brazilian Institution of Higher Education. The results showed that after the stage of culture shock, students feel more connected to local culture, make more friends and feel more confident. It was confirmed also that almost all international students managed to adapt well to local customs.
\end{abstract}

Keywords: Academic Mobility. Cultural Adaptation. International students. "U" "W" Curve. 


\section{INTRODUÇÃ̃o}

O desenvolvimento rápido das tecnologias de comunicação tem facilitado o acesso ao conhecimento, a troca de informações e estimulado o comércio entre as nações do mundo, reforçando os movimentos da globalização. Consequentemente, a migração e a movimentação de pessoas através das fronteiras também foram estimuladas, gerando mudanças comportamentais, culturais e sociais, e tornando cada vez mais necessário o estudo e o entendimento da diversidade cultural do planeta.

Nesse cenário de mobilidade, o Brasil apresenta-se entre os países com fortes atrativos para a migração de pessoas de outras nacionalidades. O número de cidadãos estrangeiros que solicitaram refúgio ao governo brasileiro cresceu nos últimos três anos em 254\%. Em 2010, foram feitas 566 solicitações à Polícia Federal e, em 2012, esse número saltou para 2.008 pessoas (CHAGAS, 2013).

A existência de certa curiosidade sobre o Brasil, as oportunidades de emprego e estudo, assim como a qualidade do ensino, instigaram também a vinda de um crescente número de estudantes estrangeiros ao país em 2012 (GIRALDI, 2013). Colombianos, portugueses, franceses e angolanos são os que mais procuram o território brasileiro para estudar, segundo o Ministério das Relações Exteriores do Brasil. Em 2012, 1.333 estudantes colombianos, 944 portugueses, 934 franceses e 745 angolanos vieram para o Brasil. Comparado a 2011, o número de colombianos interessados em estudar no Brasil cresceu em quase 50\% (GIRALDI, 2013).

$\mathrm{Na}$ observância desses movimentos migratórios, quando essas pessoas se deslocam para viver em outro país, seja com o intuito de estudar, trabalhar ou morar, as diferenças culturais são percebidas, mesmo que de formas diferentes por cada indivíduo, influenciando na sua adaptação cultural. Isto ocorre, pois o estrangeiro, ao migrar para outro país, traz consigo suas próprias referências culturais, que envolvem hábitos, crenças, valores, costumes, idioma, história, forma de se relacionar, entre outras.

A análise da adaptação cultural dos indivíduos sob o ponto de vista da teoria da curva "U" e da curva "W" tem facilitado o entendimento desse processo e subsidiado cientificamente a compreensão, orientando sobre o tipo de apoio que pode ser dado para minimizar os impactos sofridos durante o processo de adaptação, especialmente aos estudantes internacionais em mobilidade. 
Mesmo que a procura pelo Brasil como local para estudar, trabalhar ou morar esteja cada vez maior, buscou-se, para esta pesquisa, focar na análise da adaptação cultural dos estudantes internacionais, uma vez que é a modalidade que mais tem apresentado crescimento nos últimos anos, gerando a necessidade de rápidas mudanças e adaptações por parte da sociedade.

Por essa razão, analisar a adaptação cultural, sob o ponto de vista das teorias da curva "U” e da curva "W" está no cerne desta investigação. Analisam-se os diferentes momentos de vivências de estudantes internacionais, numa Instituição de Ensino Superior, localizada na região nordeste do Estado do Rio Grande do Sul, que já conta com uma expressiva população de estudantes provenientes de vários países. A Instituição, por questões legais e para resguardo dos alunos, não autorizou o repasse de informações pessoais, mas informou os contatos de estudantes que já retornaram aos seus países de origem. Por esta razão, a pesquisa foi realizada somente com estudantes provenientes do Exterior, que estão ou estiveram no Brasil, os quais serviram de objeto de análise para este estudo.

Os resultados mostram que, após a fase inicial do choque cultural, os estudantes estão mais conectados à cultura local, fazem mais amizades e se sentem mais confiantes. É possível afirmar, também, que quase todos os estudantes internacionais, apesar de dificuldades, conseguiram se adaptar aos costumes locais.

\section{REFERENCIAL TEÓRICO}

\subsection{MOBILIDADE ACADÊMICA}

A mobilidade acadêmica existe desde o século XVII, quando estudantes europeus faziam intercâmbios de estudo (TEICHLER, 2003) e é considerada um dos fenômenos que mais cresce no Ensino Superior no século XXI. Mais de três milhões de estudantes estão atualmente se mobilizando e cruzando fronteiras geográficas, culturais, digitais e educacionais, na busca de uma educação internacional (BAHNDARI; BLUMENTHAL, 2011).

Esta mobilidade para distintas Instituições de Ensino Superior serve para aumentar a consciência mundial dos estudantes, obter conhecimento inestimável e vivenciar uma experiência única, repleta de entendimento e conhecimento sobre as inumeráveis diferenças comportamentais e culturais. A mobilidade crescente retrata o interesse cada vez maior dos estudantes em se prepararem para fazer parte de um mundo interdependente. A experiência 
internacional e o conhecimento gerado através dela têm causado efeitos ideológicos nos governos e empregadores do mundo inteiro, que não somente valorizam, mas também vêem a necessidade de ter especialistas bem formados e globalmente informados, para poder consolidar sucesso nacional, regional e individual (UNESCO).

Nos anos 90, a evolução da mobilidade acadêmica internacional levou à mudança dos conceitos da mesma. A UNESCO passou a separar a mobilidade em duas categorias: Vertical Mobility (mobilidade vertical) e Horizontal Mobility (mobilidade horizontal). A distinção decorre das diferenças econômicas dos países envolvidos na mobilidade acadêmica (STALLIVIERI, 2009).

Assim, a mobilidade vertical implica o deslocamento de estudantes procedentes de países economicamente desfavorecidos para países economicamente mais fortes, portanto, oportunizando o acesso a instituições e, consequentemente, programas superiores em termos de qualidade educacional, resultando no acréscimo de valor aos estudantes nos seus respectivos mercados de trabalho. Geralmente, a mobilização vertical pressupõe que o estudante estrangeiro realize o curso acadêmico inteiro na instituição anfitriã. Portanto, já que a conclusão do curso no Exterior implica um período de duração maior fora do país de origem, a permanência do estudante no país hospedeiro se torna mais fácil de acordo com o grau de adaptação do individuo às diferenças enfrentadas, tanto no país quanto na instituição anfitriã. Segundo a UNESCO, esse tipo de mobilidade é a mais escolhida entre os acadêmicos internacionais (STALLIVIERI, 2009).

Frente a isso, é natural acreditar que estudar no Exterior está se tornando mais fácil, e até mesmo mais comum. No entanto, os estudantes internacionais ainda representam uma minoria entre as populações estudantis, uma vez que a mobilidade não está disponível para todos. Enquanto para alguns uma experiência no Exterior será uma escolha óbvia, para outros será apenas um impossível sonho (BYRAN; DERVIN, 2008).

Dessa forma, a fim de promover a vivência internacional, através da mobilidade acadêmica para estudantes que não têm essa possibilidade, o Brasil oferece oportunidades de formação superior a cidadãos de países em desenvolvimento com os quais mantém acordos educacionais e culturais, como por exemplo, através do PEC-G (Programa de EstudantesConvênio de Graduação) (MEC, 2013), que traz, especialmente, estudantes africanos e latinoamericanos ao Brasil. 


\section{ANÁLISE DA ADAPTAÇÃO CULTURAL DE ESTUDANTES INTERNACIONAIS SOB O PONTO DE \\ VISTA DAS TEORIAS DA CURVA "U" E DA CURVA "W" \\ DOI: http://dx.doi.org/10.5007/1983-4535.2015v8n3p26}

Independentemente da circunstância que leva o estrangeiro à mobilidade acadêmica, todos os indivíduos sentirão, com maior ou menor intensidade, o impacto das diferenças culturais, já que todo país tem suas próprias características culturais.

\subsection{ADAPTAÇÃO CULTURAL}

Peterson (2004) define cultura como o conjunto relativamente estável de valores e crenças internas, geralmente realizadas por grupos de pessoas em países ou regiões, e o impacto perceptível que esses valores e crenças têm sobre os povos em relação aos seus comportamentos visíveis e seu ambiente. Baseado nos estudos realizados em 1976, por Edward Hall, que desenhou o cultural iceberg, Peterson (2004) usou a mesma analogia para descrever cultura: ele a descreve como um iceberg que possui uma parte que pode ser vista (acima da linha da água), e outra que não pode ser vista (abaixo da linha da água). Na ponta do iceberg encontra-se tudo que pode ser percebido com os cinco sentidos: idioma, arquitetura, comida, população, música, vestuário, arte e literatura, ritmo de vida, gestos, atividades de lazer e esportes. A parte inferior do iceberg determina valores, tais como: noções de tempo, como o indivíduo se encaixa na sociedade, crenças sobre a natureza humana, regras sobre relacionamentos, motivações para sucesso, papel de adultos e crianças dentro da família, a tolerância para a mudança, comportamento machista, importância das expressões faciais, harmonia, sistemas de liderança, de preferência, estilos de comunicação, entre outros.

Para Hofstede (2010), cultura é sempre um fenômeno coletivo, porque é, pelo menos parcialmente, compartilhada com as pessoas que vivem ou viveram no mesmo ambiente social. Assim, a cultura é adquirida e não herdada, na qual boa parte foi aprendida na infância, uma vez que provém do ambiente social em que o indivíduo está inserido. Cultura consiste nas regras não escritas do jogo social e engloba os padrões de pensamento, de sentimentos e de ação, designando-os como programações mentais. Estas são resultado da aprendizagem contínua do ser humano através da assimilação, que uma vez instaladas na mente, é necessário desaprendê-las antes de aprender algo diferente.

A cultura pode ser vista como um fenômeno coletivo que é partilhado por um grupo social, uma nação ou uma comunidade. Também é um conjunto de crenças e valores que definem as características e a conduta de um grupo étnico ou uma nação, estando em permanente processo de mudança. Deste modo, a adaptação de um indivíduo numa outra 


\section{ANÁLISE DA ADAPTAÇÃO CULTURAL DE ESTUDANTES INTERNACIONAIS SOB O PONTO DE \\ VISTA DAS TEORIAS DA CURVA "U" E DA CURVA "W" \\ DOI: http://dx.doi.org/10.5007/1983-4535.2015v8n3p26}

cultura é um processo, e como tal passa por várias etapas. Esta inserção, chamada adaptação cultural, é um processo no qual o indivíduo tem a habilidade de eficientemente funcionar em uma cultura distinta da que ele ou ela foi originalmente socializado (HASLBERGER, 2005).

Assim, a adaptação cultural refere-se a mudanças que acontecem com os indivíduos em resposta às necessidades do ambiente a que eles estão inseridos. A adaptação cultural pode ser dividida em fases.

\subsection{FASES DA ADAPTAÇÃO CULTURAL DA TEORIA DA CURVA "U”}

Pessoas que residem em um ambiente cultural estrangeiro relatam mudanças de sentimentos ao longo do tempo de permanência no Exterior. Lysgaard (1955) aprofundou os estudos sobre esta temática e desenvolveu a teoria da curva "U" a partir de uma análise sobre a vivência de estudantes noruegueses nos Estados Unidos. Outros autores, como Joly (1992) e Hofstede (2010) também introduziram suas teorias sobre as fases da adaptação cultural com nomenclaturas e fases diferentes, no entanto, também correspondem à curva "U" de Lysgaard (1995).

Hofstede (2010) chama a atenção para o tempo que modifica em cada fase, pois existem estrangeiros que ficam por um curto período de até três meses e experimentam fases de euforia, choque cultural e estabilidade em um mesmo período. O Quadro 1 mostra um resumo das fases de adaptação cultural.

\begin{tabular}{|c|c|c|}
\hline Adaptação cultural & Fases & Autor \\
\hline \multirow{4}{*}{$\begin{array}{l}\text { Um processo que, apesar } \\
\text { de não necessariamente } \\
\text { invariável, geralmente } \\
\text { progride através de } \\
\text { quatro etapas básicas, } \\
\text { em ordem, e com as suas } \\
\text { próprias características }\end{array}$} & $\begin{array}{l}\text { Lua de Mel: excitação e euforia, onde tudo sobre o país estrangeiro } \\
\text { parece novo e intrigante. }\end{array}$ & \multirow{4}{*}{ Oberg (1954) } \\
\hline & $\begin{array}{l}\text { Choque Cultural: constatação de que existem muitas diferenças } \\
\text { culturais que tinham sido previamente escondidas ou ignorados em } \\
\text { prol de semelhanças aparentes. Esta fase se manifesta tanto através } \\
\text { de sintomas físicos, quanto emocionais, e resulta das situações } \\
\text { confusas e ambíguas. }\end{array}$ & \\
\hline & $\begin{array}{l}\text { Adaptação ou Assimilação à Cultura-Alvo: não há mais problema } \\
\text { com a proficiência na língua-alvo nem de adaptação. }\end{array}$ & \\
\hline & $\begin{array}{l}\text { Domínio: estágio onde o domínio do ambiente está completo, } \\
\text { adquirindo uma segunda identidade. }\end{array}$ & \\
\hline \multirow{4}{*}{$\begin{array}{l}\text { Adaptação cultural como } \\
\text { um processo }\end{array}$} & Fase do Encantamento: surpresas com as novidades do novo país. & \multirow{4}{*}{ Joly (1992) } \\
\hline & $\begin{array}{l}\text { Negativismo Extremo: o estrangeiro sente dificuldades de se } \\
\text { expressar, se sente frustrado e percebe as diferenças entre os países, } \\
\text { como: diferenças no humor, gestos, graus de intimidade, e ritmos } \\
\text { mais lentos ou mais rápidos em relação ao que estava acostumado. }\end{array}$ & \\
\hline & $\begin{array}{l}\text { Distância ou Integração: marcado pela volta do estrangeiro ao país } \\
\text { de origem ou não. A escolha é resultado da forma que o estrangeiro } \\
\text { enfrentou a fase anterior. }\end{array}$ & \\
\hline & $\begin{array}{l}\text { Choque da volta: quem retorna ao país de origem já não é mais o } \\
\text { mesmo e, consequentemente, sofre um novo choque cultural. }\end{array}$ & \\
\hline
\end{tabular}




\section{ANÁLISE DA ADAPTAÇÃO CULTURAL DE ESTUDANTES INTERNACIONAIS SOB O PONTO DE \\ VISTA DAS TEORIAS DA CURVA "U" E DA CURVA "W" \\ DOI: http://dx.doi.org/10.5007/1983-4535.2015v8n3p26}

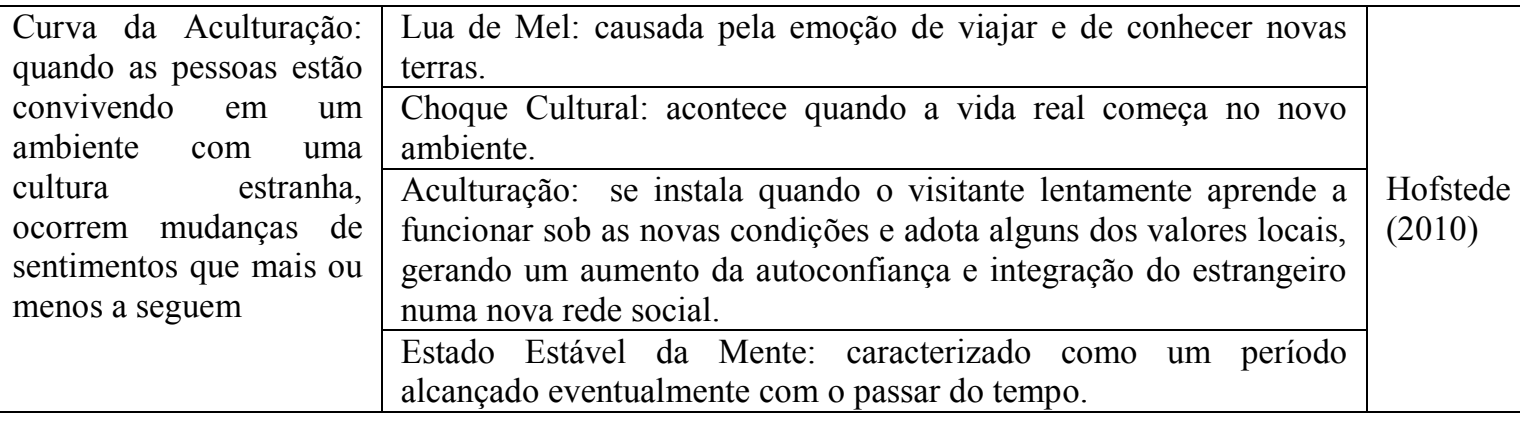

Hofstede

(2010)

Quadro 1 Fases da adaptação cultural

Fonte: Autores

\subsection{ABORDAGEM DE ADAPTAÇÃO CULTURAL DA TEORIA DA CURVA “W”}

Para Gullahorn e Gullahorn (1963) a curva "W" é uma segunda curva "U", adicionando o choque cultural reverso que se dá pela experiência de um indivíduo ao retornar à sua cultura de origem.

De acordo com Gullahorn e Gullahorn (1963), a principal diferença entre choque cultural reverso e choque cultural é a expectativa dos viajantes. Estrangeiros frequentemente esperam retornar a uma casa inalterada, com pessoas também inalteradas, o que não é o caso. Em outras palavras, uma pessoa pode já estar preparada para as diferenças culturais quando entrar em uma nova cultura, minimizando potencialmente os efeitos de choque cultural. A teoria também diz que as expectativas do regresso influenciam negativamente no choque cultural reverso. Neste caso, o viajante não espera enfrentar dificuldades, já que está voltando para casa, e pensa que vai encontrar amigos, familiares e cultura inalterada (GAW, 2010). Hoffenburger, Mosier e Stokes (1999) adaptaram a curva "U" para a curva "W", para estudantes internacionais convivendo em um novo ambiente cultural, conforme o Quadro2.

\begin{tabular}{|c|c|c|}
\hline FASE & PERÍODO & CARACTERÍSTICA \\
\hline Lua de Mel & $\begin{array}{l}\text { Antes da chegada dos estudantes à universidade e } \\
\text { durante os primeiros dias após a chegada. }\end{array}$ & $\begin{array}{l}\text { Entusiasmo pelo novo } \\
\text { (pessoas, cultura;...). }\end{array}$ \\
\hline $\begin{array}{l}\text { Choque } \\
\text { cultural }\end{array}$ & $\begin{array}{l}\text { Dificuldade do estudante internacional de se adaptar a } \\
\text { um novo conjunto de ambientes. Pode durar de três a } \\
\text { seis meses após a chegada. }\end{array}$ & $\begin{array}{l}\text { Expectativa não atendida; } \\
\text { sensação de frustração. }\end{array}$ \\
\hline $\begin{array}{l}\text { Adaptação } \\
\text { inicial }\end{array}$ & $\begin{array}{l}\text { Adaptação física dos estudantes e pelo sentimento de } \\
\text { estar mais "em casa" na universidade que pode levar } \\
\text { entre sete e nove meses. }\end{array}$ & 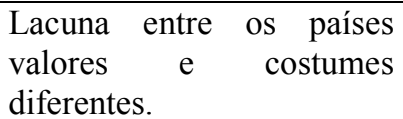 \\
\hline $\begin{array}{l}\text { Isolamento } \\
\text { mental }\end{array}$ & $\begin{array}{l}\text { Adaptação ao ambiente físico, mas as relações ainda } \\
\text { são difíceis pelas diferenças culturais. Melhorias } \\
\text { percebidas após dez a doze meses de permanência. }\end{array}$ & Solidão. \\
\hline $\begin{array}{ll}\text { Aceitação } & \text { e } \\
\text { integração }\end{array}$ & $\begin{array}{l}\text { Os alunos sentem que fazem parte do novo ambiente } \\
\text { ou da nova comunidade. São necessários, pelo menos, } \\
\text { doze meses para atingir esta fase de aceitação e de } \\
\text { integração. }\end{array}$ & Integração, \\
\hline
\end{tabular}

Quadro 2 Adaptação da curva "U" para curva "W" para estudantes internacionais Fonte: Baseado em Hoffenburger, Mosier e Stokes (1999). 
Para Hotolla (2004) o uso das teorias das curvas "U" e "W" não pode ser visto como uma explicação aceitável para a adaptação cultural, pois é uma descrição idealizada da realidade.

A duração das fases individuais pode variar de pessoa para pessoa, mas de modo geral, pesquisas sugerem que a medida, direção, magnitude e duração da dinâmica das fases da adaptação cultural têm um impacto significativo sobre a vida em um novo ambiente, especialmente durante o primeiro ano (KEALEY; RUBEN, 1979).

\subsection{CHOQUE CULTURAL}

Estudos realizados mostram que dentro das fases do processo de adaptação cultural que os estrangeiros passam, a fase mais crítica do processo é a denominada de choque cultural (KATO; PEREIRA; PIMENTEL, 2005).

Pedersen (1995) define choque cultural como uma profunda experiência pessoal que não afeta todas as pessoas da mesma forma e nem mesmo a mesma pessoa de igual maneira quando acontece novamente. Velásquez (2009) descreve choque cultural como um desconforto emocional e até mesmo físico que as pessoas sentem quando tem de deixar tudo familiar para trás e tem que encontrar seu caminho numa nova cultura que possui um jeito diferente de viver e uma mentalidade distinta. Hofstede (2010) acrescenta que geralmente o estrangeiro experimenta algum tipo de choque cultural quando é inserido num novo ambiente cultural.

Até recentemente, choque cultural era considerado uma experiência negativa. Oberg (1960) mencionou seis aspectos negativos do choque cultural, incluindo: (i) tensão resultante do esforço na adaptação psicológica; (ii) um sentimento de perda ou de privação referindo-se à perda de família, amigos antigos, status, cargo, e/ou propriedades; (iii) rejeição da, ou pela, nova cultura; (iv) confusão na definição do lugar, expectativas, sentimentos e identidade própria; (v) ansiedade inesperada, nojo, ou indignação sobre as diferenças culturais entre os antigos e os novos caminhos; e (vi) sentimentos de impotência como resultado de não lidar bem no novo ambiente.

Explicações mais recentes sobre choque cultural descrevem que o período de ajustamento é uma situação de crescimento e desenvolvimento, na qual por mais doloroso que seja, pode resultar em introspecções positivas e até mesmo essenciais (PEDERSEN, 1995). Velásquez (2009) explica que superar o choque cultural leva tempo, passa por diversas fases e 
não pode ser superado da noite para o dia. De acordo com Bochner e Furnham (1982 apud UWAJE, 2009), os indivíduos, sofrendo de choque cultural, experimentam um impacto de maneiras e formas diferentes e, portanto, apresentam sintomas diversos.

O Quadro 3 apresenta um resumo dos fatores influenciadores do choque cultural do estrangeiro quando inserido em uma nova cultura e que foram perpassados até aqui no desenvolvimento da revisão bibliográfica.

\begin{tabular}{|c|c|c|}
\hline FATORES & DESCRIÇÃO & AUTOR \\
\hline $\begin{array}{l}\text { Experiência } \\
\text { internacional } \\
\text { anterior }\end{array}$ & $\begin{array}{l}\text { Supõe a existência de uma influência que afeta como o indivíduo } \\
\text { lida com o choque cultural e como ele se adapta a uma nova } \\
\text { cultura (experiência tende a reduzir o choque). }\end{array}$ & $\begin{array}{l}\text { Parker; Mcevoy (1993); } \\
\text { Mcleod (2008) }\end{array}$ \\
\hline $\begin{array}{l}\text { Diferenças } \\
\text { culturais }\end{array}$ & $\begin{array}{l}\text { A "distância cultural" ou "resistência cultural", quanto mais } \\
\text { diferente a cultura do país anfitrião for do país de origem, é mais } \\
\text { provável que o processo de adaptação seja mais difícil. }\end{array}$ & $\begin{array}{l}\text { Marker; Mcevoy (1993); } \\
\text { Searle; Ward (1990) }\end{array}$ \\
\hline $\begin{array}{l}\text { Fluência no } \\
\text { idioma }\end{array}$ & $\begin{array}{l}\text { Maior interação com a comunidade do país hospedeiro melhora o } \\
\text { desempenho na língua-alvo (quanto maior o nível de fluência e } \\
\text { conforto ao falar o idioma, menor será o grau do choque cultural). }\end{array}$ & $\begin{array}{l}\text { Kennedy; Kard (1992); } \\
\text { Mcleod (2008); } \\
\text { Gibson (2000) }\end{array}$ \\
\hline $\begin{array}{l}\text { Tempo } \\
\text { estadia }\end{array}$ & $\begin{array}{l}\text { Quanto maior for o tempo de residência no país hospedeiro, mais } \\
\text { positiva será a atitude, interação, adaptação e realização do } \\
\text { estrangeiro }\end{array}$ & Lysgaard (1955) \\
\hline $\begin{array}{l}\text { Imagem } \\
\text { percebida do } \\
\text { país destino }\end{array}$ & $\begin{array}{l}\text { Se a imagem percebida realmente corresponde ao que o país ou } \\
\text { região representa, o choque cultural será mais ameno, caso } \\
\text { contrário mais intenso. }\end{array}$ & $\begin{array}{l}\text { (Anholt, 2000); (Del Rio, } \\
\text { 1992); (Barich; Kotler, } \\
\text { 1991; Heslop et al., 2008) }\end{array}$ \\
\hline
\end{tabular}

Quadro 3 Fatores influenciadores do choque cultural

Fonte: Autores

\section{MÉTODO}

O método utilizado para este estudo foi a pesquisa descritiva com abordagem quantitativa. A pesquisa descritiva é um tipo de pesquisa conclusiva que tem como principal objetivo a descrição detalhada e supõe que o pesquisador tenha conhecimento anterior sobre o problema que é o pano de fundo deste estudo. Segundo Roesch (1999), quando o propósito do estudo é obter informações sobre determinada população, como e em que proporção seus membros têm certa opinião ou característica, ou com que frequência certos eventos estão associados entre si, como se objetivou nesta pesquisa.

\subsection{INSTRUMENTO DE COLETA DE DADOS E AMOSTRA}

Segundo Malhotra et al. (2005), levantamento de campo (survey) é uma forma de coleta de dados confiável, simples e fácil, que se faz através da aplicação de questionários. Surveys tornam a análise de informações e dados obtidos mais precisa, pois tem a característica de obter informações em forma de números (HAIR, 2005). 


\section{ANÁLISE DA ADAPTAÇÃO CULTURAL DE ESTUDANTES INTERNACIONAIS SOB O PONTO DE \\ VISTA DAS TEORIAS DA CURVA "U" E DA CURVA "W" \\ DOI: http://dx.doi.org/10.5007/1983-4535.2015v8n3p26}

Existem dois tipos de classificação para questionários: estruturado ou não estruturado e questões fechadas ou questões abertas (HAIR, 2005). Neste trabalho foram utilizadas questões fechadas, ou estruturadas, permitindo que o entrevistado respondesse alternativas já elaboradas, assim eliminando a possibilidade de responder com as próprias palavras. No questionário utilizou-se a escala de Likert, que, segundo Malhotra et al. (2005), é uma das escalas por itens amplamente utilizada. Foi solicitado que estudantes internacionais indicassem seu grau de concordância ao escolher uma das cinco escalas de respostas (escala Likert de cinco pontos).

As perguntas que compuseram o questionário foram baseadas no referencial teórico e embasadas no quadro com o resumo das características das curvas "U" e "W" (Quadro 1), e os influenciadores do choque cultural (Quadro 2). As questões foram divididas em grupos a partir das características descritas pelos autores sobre as cinco fases da adaptação cultural, visto que cada fase representa três meses de estadia no Brasil, a não ser a última fase, que representa mais de doze meses no país. Por fim, foram solicitadas informações relacionadas à identificação e informações pessoais dos respondentes.

Segundo Malhotra et al. (2005, p. 261), "a população-alvo é a coleção de elementos ou objetos que possuem a informação que o pesquisador está buscando". A população-alvo desta pesquisa consiste em estudantes internacionais que estejam estudando ou que tenham estudado numa Instituição de Ensino Superior. Segundo a Instituição, o número de estudantes internacionais no período de 2009 e 2013 foi de 459, contemplando somente estudantes de graduação, ou seja, PMAI (Programa de Mobilidade Acadêmica Internacional) e PEC-G (Programa de Estudantes-Convênio de Graduação).

A técnica de amostragem escolhida foi a não probabilística por conveniência, ou seja, os que aceitaram responder. Os questionários foram enviados por e-mail e pelas redes sociais para um total de 137 pessoas. Dentre os questionários enviados, 48 estudantes responderam. Não foi possível enviar os questionários para os 459 estudantes, já que a Instituição não autorizou a disponibilização dos contatos por questões de privacidade dos alunos. Alguns contatos foram disponibilizados por entidades que conhecem os estrangeiros e que providenciaram as informações. Outros contatos foram obtidos através dos próprios estudantes internacionais e, por fim, outros foram disponibilizados pela Instituição, no entanto, somente estudantes que já retornaram para seus países de origem. 
O procedimento para análise das respostas obtidas do questionário foi através de uma análise quantitativa de caráter descritivo, podendo-se assim calcular as médias, computar porcentagens, examinar os dados para verificar se possuem significância estatística e correlacionar variáveis (ROESCH, 1999).

Foi utilizado o software para análise estatística de dados SPSS (Statistical Package for the Social Sciences-Pacote Estatístico para as Ciências Sociais), versão 21.

\section{ANÁLISE E DISCUSSÃo DOS RESULTADOS}

Esta seção tem como objetivo apresentar os resultados obtidos a partir da análise do questionário entregue aos estudantes internacionais que vivem ou que viveram no Brasil. $\mathrm{Na}$ primeira parte, apresenta-se o perfil dos respondentes. Na sequência, são analisados os resultados obtidos das questões relacionadas ao período de estadia no Brasil junto às fases da adaptação cultural.

\subsection{PERFIL DOS RESPONDENTES}

Dentre os 48 estudantes internacionais respondentes do questionário, 26 são do sexo feminino e 22 são do masculino, representando percentual de 54,2\% e 45,8\% respectivamente.

A maioria dos participantes apresentou idade entre 22 e 24 anos, correspondendo a $47,9 \%$ do total. A amostra da pesquisa compõe-se de estudantes internacionais de 15 países, sendo que os países com predominância de respondentes são a Espanha, em segundo lugar a França e em terceiro a Argentina (Figura 1 ).

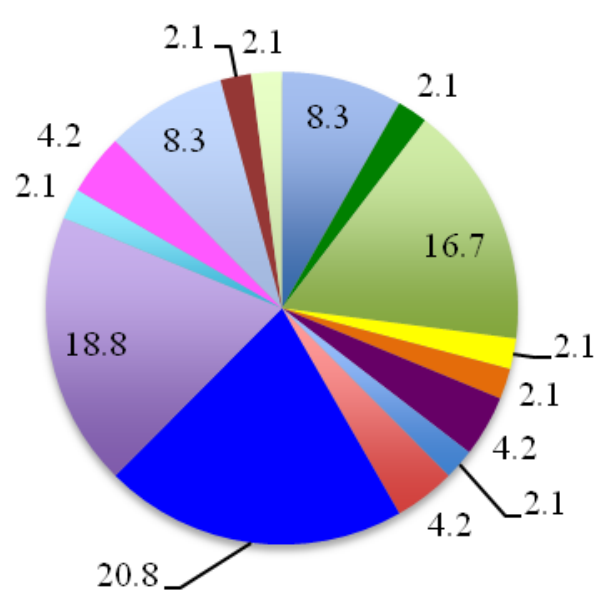

$$
\begin{aligned}
& \text { - Alemanha } \\
& \text { - Angola } \\
& \text { - Argentina } \\
& \text { Canadá } \\
& \text { China } \\
& \text { - Colombia } \\
& \text { - Costa Rica } \\
& \text { Equador } \\
& \text { - Espanha } \\
& \text { - França } \\
& \text { - Guiné-Bissau } \\
& \text { Itália } \\
& \text { - México } \\
& \text { - Peru } \\
& \text { Portugal }
\end{aligned}
$$

Figura 1 País de ascendência dos respondentes Fonte: Autores 
O período de tempo no Brasil que mais estudantes assinalaram foi entre quatro e seis meses, representando $50 \%$ dos respondentes. Isto pode ocorrer pelo fato de que a maioria dos estudantes internacionais realizou viagens de mobilidade acadêmica pelo período de um semestre.

\subsection{ANÁLISE E DISCUSSÃO DOS RESULTADOS}

A partir das informações obtidas na análise dos resultados, observa-se que a maioria dos respondentes estava de acordo com as seis primeiras questões do questionário, que envolvem o período antes de vir ao Brasil e os três primeiros meses de estadia, comprovando a teoria proposta pelos autores durante a primeira fase da adaptação cultural. As afirmações envolviam: entusiasmo ao pensar em viajar e conhecer novas terras (Hofstede, 2010; Oberg, 1954; Joly, 1992), a sensação de que tudo era novo e intrigante (OBERG, 1954), atitudes positivas sobre o novo país (JOLY, 1992), empolgação com a possibilidade de morar com diferentes pessoas (HOFFENBURGER; MOSIER; STOKES, 1999).

A questão envolvendo a impressão do estrangeiro de que o Brasil é parecido com o próprio país de origem (OBERG, 1954) também obteve concordância dos respondentes, no entanto não tão significativa. Os estudantes argentinos foram os que mais confirmaram terem percebido poucas diferenças entre o Brasil e o seu país durante os três primeiros meses de estadia. Isto pode se dever ao fato de que o Brasil e a Argentina são países próximos geograficamente e apresentam menor distância cultural.

A segunda fase da adaptação cultural é chamada de Choque Cultural por Hofstede (2010), Oberg (1954) e Hoffenburger, Mosier e Stokes (1999), ou Negativismo Extremo, por Joly (1992). Esta fase envolve o período entre quatro e seis meses no Brasil. Dentre as fases da adaptação cultural, a mais crítica do processo é considerada a segunda fase (KATO; PEREIRA; PIMENTEL, 2005).

Foi apoiada pela pesquisa a constatação dos estudantes sobre as diferenças culturais que previamente não foram percebidas nos primeiros meses, proposto por Oberg (1954) e Joly (1992, 2000).

No entanto, a opinião dos estrangeiros com relação à distância cultural entre o Brasil e seus países de origem não foi significativa, já que as opiniões foram diversificadas. Isto ocorreu talvez devido à melhoria na qualidade das informações e ao processo de globalização atualmente existentes. Além disso, se deve provavelmente ao fato de que os respondentes 


\section{ANÁLISE DA ADAPTAÇÃO CULTURAL DE ESTUDANTES INTERNACIONAIS SOB O PONTO DE \\ VISTA DAS TEORIAS DA CURVA "U" E DA CURVA "W" \\ DOI: http://dx.doi.org/10.5007/1983-4535.2015v8n3p26}

eram de países diferentes com pontos de vista e opiniões diferentes, não sendo possível identificar se a distância cultural era expressiva ou não. De acordo com Parker e McEvoy (1993) e Searle e Ward (1990 apud MCLEOD, 2008), quanto maior for a distância cultural entre os países, mais forte é o choque cultural e mais difícil será a adaptação.

Portanto, pode-se concluir que foi significativa a percepção dos estudantes sobre as diferenças culturais entre os países, mas, não foi significativa a percepção de muitas diferenças culturais entre o Brasil e os países dos estudantes. Vale ressaltar que a maioria dos argentinos não acredita que o Brasil e a Argentina tenham muitas diferenças culturais, ao contrário dos franceses, grupo no qual quase todos afirmaram que a França e o Brasil apresentam muitas diferenças culturais.

Foi confirmada a teoria de Oberg (1954) de que os estudantes passariam por momentos confusos nos quais não saberiam como se comportar adequadamente em uma cultura estrangeira. No entanto, um dos aspectos propostos por Oberg (1954) como negativo no choque cultural, o sentimento de perda referindo-se à família e amigos antigos, não mostrou ser um fator negativo na adaptação cultural para a maioria dos estudantes.

Joly (1992) afirmou que na segunda fase da adaptação cultural o estrangeiro sente dificuldades ao se expressar e esta afirmação foi comprovada pela maioria das opiniões dos estrangeiros que afirmaram ter enfrentado dificuldades neste aspecto. No entanto, a partir das respostas obtidas, a maioria dos estudantes dessa pesquisa aparentemente não sofreu grandes dificuldades com a língua portuguesa. Kennedy e Ward (1992) e McLeod (2008) afirmaram que quanto melhor a fluência no idioma, mais fácil será a adaptação cultural. Portanto, conclui-se que a maioria dos participantes lidou com problemas para se expressar, o que é natural, mas a língua portuguesa não foi um grande obstáculo. Isto pode ser devido à proximidade da língua espanhola com a língua portuguesa, e devido ao fato de que alguns respondentes já tinham como língua-mãe o português. No entanto, até mesmo estudantes que tinham como língua-mãe o português, advindos de países como Angola, Portugal e GuinéBissau, afirmaram ter passado por dificuldades ao se expressar. Assim, foram comprovadas as teorias de Bennett (1998) e Gibson (2000), na qual pessoas de diferentes culturas são constituídas de diferentes realidades sensoriais. Os estudantes sentiram dificuldades com relação à interpretação de sinais não verbais, influenciando na adaptação cultural.

A questão que envolvia sentimento de frustração, proposta por Joly (1992), não foi conclusiva, já que as respostas foram diversificadas. Este resultado pode estar ligado à maior 


\section{ANÁLISE DA ADAPTAÇÃO CULTURAL DE ESTUDANTES INTERNACIONAIS SOB O PONTO DE \\ VISTA DAS TEORIAS DA CURVA "U" E DA CURVA "W" \\ DOI: http://dx.doi.org/10.5007/1983-4535.2015v8n3p26}

limitação do estudo que envolve o número de respondentes obtidos na pesquisa. No entanto, a mesma teoria que envolve a procura de outros estrangeiros da mesma nacionalidade para desabafar, não foi confirmada pelos respondentes. Esta alternativa é sugerida porque foi observado, a partir dos resultados, que todos, exceto um dos respondentes que concordaram totalmente ou parcialmente com a afirmação, correspondem às nacionalidades predominantes na amostra, de origem espanhola, francesa e argentina.

Com relação à autoexclusão (JOLY, 1992), sentimento de estar perdido no país (HOFFENBURGER; MOSIER; STOKES, 1999) e retorno para o país de origem (OBERG, 1954), a maioria dos estudantes não concordou com as afirmações. Com base nisso, nota-se que, nesses aspectos da fase do choque cultural, a maioria dos estudantes não enfrentou grandes dificuldades.

Uma das questões estudadas foi a influência positiva da experiência internacional anterior no choque cultural, proposta por Bochner e Furnham (1982 apud UWAJE, 2009), Parker e McEvoy (1993), McLeod (2008). No caso da presente pesquisa, a maioria dos respondentes não concordou com a influência positiva, mas deve-se ressaltar que essa discordância pode ter ocorrido pela falta de experiência internacional do respondente e não porque não considera a experiência internacional como influência positiva.

Outro fator que influencia na intensidade do choque cultural e que foi enfrentado pelos estudantes internacionais é se a imagem percebida do país antes da viagem corresponde ao que o estrangeiro encontra quando chega ao país, proposto por Del Rio (1992), Heslop et al. (2008 apud MARIUTTI, 2013), Anholt (2000). A partir das respostas obtidas, notou-se que a maioria dos estudantes veio ao Brasil imaginando o país de uma maneira e, ao chegarem, se depararam com algo não esperado. Este imprevisto pode dificultar na adaptação cultural do estrangeiro, pois o estudante espera encontrar algo, mas encontra algo diferente. Segundo Heslop et al. (2008 apud MARIUTTI, 2013), a imagem dos países é apresentada como uma dimensão que orienta o estrangeiro, fornecendo informações sobre o país para o processo de tomada de decisões. De acordo com Anholt (2000) os pontos positivos relacionados à imagem do Brasil incluem a diversidade ecológica, um clima tropical agradável, as pessoas amigáveis e alegres, o futebol e o carnaval. A partir dessa imagem criada sobre o Brasil, alguns estrangeiros trazem consigo uma imagem estereotipada sobre o país como um todo, como afirmado por Gibson (2000), mas deve-se levar em consideração que o Brasil é um país de dimensões continentais, com muitas diferenças entre as regiões. Nesta pesquisa, os estudantes 
europeus foram os que mais relataram ter uma imagem diferente sobre o Brasil antes de vir e se depararam com outra ao chegar. A partir das informações obtidas, pode-se presumir que a Europa é um local onde a imagem do Brasil é estereotipada.

A terceira fase da adaptação cultural apresenta nomenclatura diferente para cada autor. Hofstede (2010) chama de Fase de Aculturação, Oberg (1954) chama de Fase de Recuperação, Joly (1992) chama de Fase de Distância ou Integração, Hoffenburger, Mosier e Stokes (1999) chamam de Fase da Adaptação Inicial. Esta fase envolve o período entre sete e nove meses no Brasil. Durante a terceira fase foram comprovadas as afirmações de Hofstede (2010) de que quando o estrangeiro chegasse nessa fase haveria aumento da autoconfiança e integração em uma nova rede social. A afirmação de Oberg (1954) sobre o sentimento de ter duas identidades foi confirmada pela maioria dos estudantes. Já a afirmação de Oberg (1954) com relação a sentir que não pertence a nenhuma cultura não foi confirmada pela maioria dos estrangeiros. Portanto, conclui-se que alguns estrangeiros se sentiram confusos com relação as suas identidades, mas não a ponto de se sentirem frustrados com a sensação de não pertencer a nenhuma das culturas.

Para Joly (1992), essa fase é marcada pela volta do estrangeiro ao seu país de origem ou não. Os participantes da pesquisa não escolheram retornar para o país de origem, a não ser um participante do México que concordou totalmente com a afirmação.

A quarta fase da adaptação cultural, como na fase 3, também teve nomenclaturas diferentes para cada autor. Hofstede (2010) chama de Estado Estável da Mente, Oberg (1954) chama de Adaptação ou Assimilação à Cultura-Alvo, Joly (1992) chama de Choque da Volta e Hoffenburger, Mosier e Stokes (1999) chamam de Isolamento Mental. Esta fase envolve o período entre dez e doze meses de estadia no Brasil.

De acordo com Hofstede (2010), a quarta fase pode ser dividida em três outras fases. Uma delas é que o estrangeiro pode continuar negativo, se comparado ao país de origem, com a qual no caso deste estudo, a maioria dos estudantes não concordou. Ou, pode continuar tão bom quanto o início da primeira fase e neste caso, o estrangeiro é considerado bi culturalmente adaptado (HOFSTEDE, 2010; OBERG, 1954), que neste estudo houve concordância da maioria dos participantes. A última opção é que nesta fase a adaptação cultural pode ser ainda melhor que no país de origem e neste caso, é como se o estrangeiro fosse nativo do país (HOFSTEDE, 2010; OBERG, 1954), e que neste estudo apresentou 


\section{ANÁLISE DA ADAPTAÇÃO CULTURAL DE ESTUDANTES INTERNACIONAIS SOB O PONTO DE \\ VISTA DAS TEORIAS DA CURVA "U" E DA CURVA "W" \\ DOI: http://dx.doi.org/10.5007/1983-4535.2015v8n3p26}

respostas variadas. Portanto, foi possível descobrir que a maioria dos estudantes se sentiu bi culturalmente adaptado, mas não a ponto de se sentir como se fosse nativo do país.

Para a maioria dos estudantes, a proficiência na língua portuguesa não foi mais um problema, como afirmado por Oberg (1954). Somente uma pessoa da China não concordou com a afirmação. Frente a isso, deve ser considerada a grande diferença entre os idiomas que levou o respondente a esta opção de resposta.

Oberg (1954) também afirmou que um relacionamento amoroso pode influenciar no entrosamento com a nova cultura. Joly (1992) descreveu que nesta fase o retorno do estrangeiro ao seu país de origem, que já não é mais o mesmo, resulta em um novo choque cultural, pois depois de passar por diferentes experiências, situações excitantes e desafiadoras, voltar para casa pode ser algo desestimulante para o estrangeiro. No entanto, nesta pesquisa as respostas não foram significativas para ambas as afirmações dos autores, pois foram bastante diversificadas. Portanto, a teoria proposta por Gullahorn e Gullahorn (1963) de que o estrangeiro sente um choque cultural reverso ao retornar ao país de origem, não foi confirmada pela pesquisa.

O sentimento de que conhecer os outros é difícil porque ninguém se sente da mesma maneira, causando sentimento de solidão, proposto por Hoffenburger, Mosier e Stokes (1999), não foi confirmado pela maioria dos participantes da pesquisa.

A quinta fase foi descrita somente por Hoffenburger, Mosier e Stokes (1999) e denominada Fase de Aceitação e Integração. Esta fase envolve o período de pelo menos doze meses de estadia no Brasil. Com base nas informações obtidas na análise dos resultados, foram confirmadas as afirmações dos autores com relação a esta fase. Os estudantes se sentiram mais em casa nesta fase do que em qualquer outra. Os estudantes sentiram que faziam parte do novo ambiente/comunidade e passaram a fazer mais amizades dentro e fora da universidade. Também, neste estudo foi comprovada a influência do fator tempo de estadia no país hospedeiro proposto por Lysgaard (1955), que sugere que quanto maior for o tempo de residência no país hospedeiro, mais positiva será a atitude, interação, adaptação e realização do estrangeiro.

A última questão não envolveu as fases da adaptação cultural, e sim a percepção dos respondentes com relação à influência negativa da cultura de origem italiana e alemã na adaptação cultural no Estado do Rio Grande do Sul. A influência da imigração dos italianos e alemães que vieram para o Estado está presente na cultura gaúcha como um todo, através da 
gastronomia, religiosidade, vocabulário típico, música, festividades, usos e costumes, contribuindo para a formação da cultura (BRUM, 2007). Por essa razão, foram destacadas essas duas culturas no questionário, que do ponto de vista dos estudantes, resultou em discordância em relação à influência negativa dessas etnias na adaptação cultural dos estrangeiros no Rio Grande do Sul.

\section{CONSIDERAÇÕES FINAIS}

A partir do estudo realizado, pode-se observar que as fases da adaptação cultural não acontecem da mesma forma para todos os estrangeiros. Este fato é esperado, já que todas as pessoas têm seus próprios costumes, valores, comportamentos, socializações, criações, enfim, as suas próprias culturas, que podem ou não ser próximas da cultura na qual está ocorrendo a adaptação.

No entanto, foi possível constatar que a primeira fase da adaptação cultural, caracterizada como uma fase de entusiasmo e de atitudes positivas antes de viajar e durante os primeiros meses foi comprovada pelos estudantes internacionais. Esta fase, junto à fase cinco, foi a que mais obteve concordância e que se mostrou mais precisa entre as fases da adaptação cultural. A fase cinco comprova, que com o passar do tempo, o estrangeiro se sente mais inserido à cultura local, faz mais amizades e se sente mais "em casa".

Observou-se, também, que, nos primeiros meses no Brasil, os estudantes não percebem tantas diferenças culturais entre os países de origem e de destino. No entanto, essas diferenças culturais são percebidas durante a segunda fase, ou seja, após um maior contato com a cultura local.

Vale ressaltar que pouca ou muita distância cultural entre os países influencia na percepção dos estudantes sobre as diferenças entre os mesmos, interferindo na adaptação. $\mathrm{O}$ mesmo ocorreu com a proximidade do idioma, que mostrou sua influência na proficiência na língua portuguesa.

Pode-se concluir que quase todos os estudantes internacionais conseguiram se adaptar bem à cultura local, pois não se sentiram "perdidos" e não se excluíram por sentir que ninguém mais sente as mesmas dificuldades. Alguns aspectos negativos do choque cultural foram confirmados, como dificuldades de se expressar, incertezas com relação a como se comportar e imagem percebida do Brasil diferente antes de chegar ao país, no entanto, não se 
mostraram como fatores que influenciaram com intensidade negativa na adaptação cultural dos estudantes.

\subsection{LIMITAÇÕES DA PESQUISA E SUGESTÕES DE ESTUDOS FUTUROS}

Esta pesquisa se limitou a questionar estudantes internacionais que tenham estudado ou que estejam estudando na Universidade de Caxias do Sul. Possivelmente, o fato de que todos os respondentes moraram ou moram em Caxias do Sul, e estudaram ou estudam na Universidade de Caxias do Sul, pode demonstrar um viés na adaptação cultural e consequentemente, na indicação de respostas dos participantes. Outra limitação enfrentada no estudo foi o número de participantes conseguidos para a pesquisa. Devido a regulamentos da Universidade de Caxias do Sul, não foi autorizado informar todos os contatos dos estudantes internacionais. Além disso, a questão do número limitado de estudantes internacionais na cidade não possibilitou a obtenção de outros contatos em outras instituições.

Outra limitação do estudo é que pode ter ocorrido certa influência na percepção da adaptação cultural pelos estudantes internacionais, causado pelos diferentes períodos de permanência dos estudantes no Brasil respondendo a uma mesma fase. Os indivíduos que estão no país por um período mais longo e que responderam a primeira fase de três meses no Brasil, por exemplo, devido a um maior contato com a cultura, por terem uma opinião diferente à do período inicial de estadia, ou por não se lembrarem exatamente como foram os primeiros meses no Brasil, podem influenciar na precisão dos resultados obtidos. De forma geral, esta limitação também ocorre para as outras fases da adaptação cultural.

No futuro, pode-se realizar uma pesquisa focada em um determinado grupo de participantes, por exemplo, todos respondentes originados de uma mesma nacionalidade. Outra possibilidade é focar a pesquisa na adaptação cultural de outros tipos de estrangeiro, como expatriados, imigrantes ou refugiados. Sugere-se a realização de uma pesquisa mais abrangente, questionando estudantes internacionais de outras universidades no Rio Grande do Sul e até mesmo no Brasil, e assim, verificar em que intensidade há diferença na percepção da adaptação cultural em outras universidades e regiões.

\section{REFERÊNCIAS}

ANHOLT, S. ANHOLT. The Nation as a Brand. Across the Board. v. 37, n. 10. The H.W. Wilson Company. N/D, 2000. Disponível em: http://www.simonanholt.com/. Acesso em: 10 jul. 2013. 
BAHNDARI, R.; BLUMENTHAL, P. International Students And Global Mobility In Higher Education: National Trends And New Directions. 1. Ed. New York: Palgrave Macmillan, 2011.

BENNETT, J. M. Basic concepts of intercultural communication. Boston: Intercultural Press, 1998.

BRUM, H. N. Regiões culturais: a construção de identidades culturais no Rio Grande do Sul e sua manifestação na paisagem gaúcha. 2007. 318 f. Tese (Mestrado em Geografia) Universidade Federal de Santa Maria, Rio Grande do Sul.

BYRAM, M.; DERVIN, F.. Students, Staff and Academic Mobility in Higher Education. UK: Cambridge Scholars Publishing, 2008. Disponível em: $<$ http://www.c-sp.org/flyers/9781847186034-sample.pdf>. Acesso em: 2 nov., 2013.

CHAGAS, M. Triplica número de estrangeiros que pedem refúgio no Brasil. Agência Brasil, 2013. Disponível em: $<$ http://agenciabrasil.ebc.com.br/noticia/2013-04-26/triplicanumero-de-estrangeiros-que-pedem-refugio-no-brasil>. Acesso em: 10 nov., 2013.

DEL RIO, V. Urban design and conflicting city images of Brazil. Special Series on Urban Design, 1992. Disponível em:

$<$ http://digitalcommons.calpoly.edu/cgi/viewcontent.cgi?article $=1004 \&$ context $=$ crp_fac $>$. Acesso em: 18 maio, 2013.

FACHIN, O. Fundamentos de metodologia. 4. ed. São Paulo: Saraiva, 2003.

FREITAS E. M. Como vivem os executivos expatriados e suas famílias? EAESP/FGV/NPP Núcleo de Pesquisas e Publicações, 2000. Disponível em:

$<$ http://gvpesquisa.fgv.br/sites/gvpesquisa.fgv.br/files/publicacoes/Rel07-2000.pdf $>$. Acesso em: 29 abril, 2013.

GAW, K. F. Reverse culture shock in students returning from overseas. International Journal of Intercultural Relations, v. 24, p. 83-104, 2000.

GIBSON, R. Intercultural Business Communication. Berlin: Cornelsen \& Oxford Press GmbH \& Co., 2000.

GIRALDI, R. Oportunidade de emprego motiva estudantes estrangeiros a virem ao Brasil. Agência Brasil, 2013. Disponível em: $<$ http://agenciabrasil.ebc.com.br/noticia/201302-11/oportunidade-de-emprego-motiva-estudantes-estrangeiros-virem-ao-brasil $>$.Acesso em: 10 nov., 2013.

GULLAHORN, J. E., and GULLAHORN J. T. An Extension of the U-Curve Hypothesis. Journal of Social Issues 19:33-47, 1963.

HALL, E. T. Beyond culture. New York: Anchor Books, 1989. 
HASLBERGER, A. Facets and dimensions of cross-cultural adaptation: refining the tools. Personnel Review, Austria, v. 34, n. 1, p. 85-109, 2005.

HESLOP L.; LUK P.; NADEAU J.; O'REILLY N. Destination in a country image context. Annals of Tourism Research, Canada, v. 35, n. 1, p. 84-106, 2008.

HOFFENGURGER K.; MOSIER, R.; STOKES B. The w-curve hypothesis model Gullahorn and Gullahorn, 1999. Disponível em: <

http://www.uwsuper.edu/fye/parents/upload/W-Curve.pdf $>$. Acesso em: 2 set, 2013.

HOFSTEDE, G. H.; HOFSTEDE, G. J.; MINKOV, M. Cultures and organizations: software of the mind. 3.ed. United States of America: Mc Graw Hill, 2010.

HOTOLLA, P. Culture Confusion: Intercultural adaptation in tourism. Annals of Tourism Research, Finland, v. 31, n. 2, p. 447-466, 2004.

HUMPFREY, S. L. Foreign student adjustment at Iowa State University. 1990. Thesis (Arts Masters) - Iowa State University.

JOLY, A. (1992). “Alteridade: ser executivo no exterior”, in: CHANLAT, J. F.(org.). O indivíduo na organização: dimensões esquecidas. São Paulo, Atlas, (p. 83-124)

KATO, H. T.; PEREIRA N. A. F.; PIMENTEL, R. Expatriação e estratégia internacional: o papel da família como fator de equilíbrio na adaptação do expatriado. RAC, v. 9, n. 4, 2005.

KEALEY J. D.; RUBEN D. B. Behavioral assessment of communication competency and the prediction of cross-cultural adaptation. International Journal of Intercultural Relations. v. 3, p. 15-47, 1979.

KENNEDY, A.; WARD C.. Locus of control, mood disturbance, and social difficulty during cross-cultural transitions. International Journal of Intercultural Relations, v. 16, p. 175194, 1992.

KOTLER, P. Administração de marketing: análise, planejamento, implementação e controle. 5 ed. São Paulo: Atlas, 1998.

LYSGAARD, S. 1955. Adjustment in a Foreign Society: Norwegian Fulbright Grantees Visiting the United States. International Social Science Bulletin 7:45-51.

MALHOTRA, N. K. [et al.]. Introdução à pesquisa de marketing. 1 ed. São Paulo: Prentice Hall, 2005.

MARIUTTI, F. G. The image of Brazil as a tourism destination: an exploratory study of the American market. International Journal of Business Administration, v. 4, n. 1, 2013.

MEC (Ministério da Educação). PEC-G. Disponível em:

http://portal.mec.gov.br/index.php?Itemid=530\&id=12276\&option=com_content\&view=artic le. Acesso em: 10 nov., 2013. 
MCEVOY, G. M.; PARKER, B. Initial examination of a model of intercultural adjustment. International Journal of Intercultural Relations, v. 17, p. 355-379, 1993.

MCLEOD, K. D. A qualitative examination of culture shock and the influential factors affecting newly-arrived Korean students at Texas a\&m university. 2008. 318 p. Dissertation (Doctorate in Philosophy) - Texas A\&M University. Disponível em: $<$ http://repository.tamu.edu/bitstream/handle/1969.1/ETD-TAMU-2707/MCLEOD DISSERTATION.pdf? sequence=1>. Acesso em: 15 junho, 2013.

OBERG, K. 1954. Culture Shock. Panel discussion at the Midwest regional meeting of the Institute of International Education in Chicago, November 28, 1951.

PEDERSEN, P.; The five stages of culture shock: critical incidents around the world. United States of America: Greenwood Publishing Group, Inc., 1995. Disponível em:

$<$ http://books.google.com.br/ >. Acesso em: 4 junho, 2013.

PETERSON, B. Cultural intelligence: a guide to working with people from other cultures. Boston: Intercultural Press, 2004.

ROESCH, S. M. A. Projetos de estágio e de pesquisa em administração: guia para estágios, trabalhos de conclusão, dissertações e estudos de caso. 2 ed. São Paulo: Atlas, 1999.

SHEN, H.; YU, B. Predicting role of linguistic confidence, integrative motivation and second language proficiency on cross-cultural adaptation. International Journal of Intercultural Relations, v. 36, p. 72-82, 2012.

STALLIVIERI, L. As dinâmicas de uma nova linguagem intercultural na mobilidade acadêmica internacional. 2009. 234 f. Tese (Doutorado em Línguas Modernas) Universidad Del Salvador, Buenos Aires, Argentina.

TEICHLER, Ulrich. Mutual recognition and credit transfer in Europe: experience and Problems. Journal of Studies in International Education, v. 7, n. 4, Winter, 2003.

UNESCO. Educação superior: reforma, mudança e internacionalização. Anais. Brasília: Unesco Brasil; Sesu, 2003. Acesso em: 25 abr. 2013

UWAJE, A. C. Culture shock, re-integration and re-entry culture shock: managing cultural differences, 2009. Disponível em $<$ http://www.munich-businessschool.de/intercultural/index.php/Culture_shock,_Re-Integration_and_Re Entry_culture_shock_-_Managing_Cultural_Differences $>$. Acesso em: 4 junho, 2013. 